\title{
Development and Validation of Highly Selective LC-MS/MS Method for the Simultaneous Estimation of Pioglitazone, Hydroxy Pioglitazone and Glimepiride in in Rat Plasma
}

\author{
Kalyani Lingamaneni* and Mukthinuthalapati Mathrusri Annapurna \\ Department of Pharmaceutical Analysis and Quality Assurance, Gandhi Institute \\ of Technology and Management (Deemed to be University), GITAM Institute of \\ Pharmacy, Visakhapatnam, Andhra Pradesh, India \\ *Corresponding Author: Kalyani Lingamaneni, Department of Pharmaceutical \\ Analysis and Quality Assurance, Gandhi Institute of Technology and Management \\ (Deemed to be University), GITAM Institute of Pharmacy, Visakhapatnam, Andhra \\ Pradesh, India.
}

Received: July 30, 2021

Published: August 04, 2021

(C) All rights are reserved by Kalyani

Lingamaneni and Mukthinuthalapati

Mathrusri Annapurna.

\section{Abstract}

The rapid, sensitive, selective and precise liquid chromatography-electrospray ionization-tandem mass spectrometry method (LC-ESI-MS/MS) for the simultaneous estimation and pharmacokinetic investigation of Pioglitazone, Hydroxy pioglitazone (M-IV), a key metabolite of pioglitazone; and glimepiride in rat plasma has been developed and fully validated. Pioglitazone and Glimepiride exert synergistic effects on blood glucose control, were investigated in rat plasma using Rosiglitazone as internal standards (IS). Solid phase extraction was carried out on $0.32 \mathrm{~mL}$ of rat plasma using Strata-X $33 \mathrm{~mm}$ polymeric sorbent cartridges, and chromatographic separation was performed on an Agilent Zorbax SB C18 column (50 mm x $4.6 \mathrm{~mm}, 3.5 \mu \mathrm{m}$ ) using a mobile phase consisting of an 80:20 (v/v) mixture of acetonitrile and 10mM ammonium formate ( $\mathrm{pH}: 3.0 \pm 0.05)$ at a flow rate of $0.8 \mathrm{~mL} / \mathrm{min}$. To quantify Pioglitazone, Hydroxy pioglitazone, Glimepiride and IS multiple reaction monitoring (MRM) transitions of $\mathrm{m} / \mathrm{z} 357.0 \rightarrow 134.1, \mathrm{~m} / \mathrm{z} 373.0$ $\rightarrow 150.1, \mathrm{~m} / \mathrm{z} 491.2 \rightarrow 352.2$ and $\mathrm{m} / \mathrm{z} 358.3 \rightarrow 135.2$ respectively were performed in positive mode. The total run time was $3.5 \mathrm{~min}$ and the elution time was about $2.8 \mathrm{~min}$. The method exhibited good separation of analytes, without interference from endogenous substances. The method has been validated according to the USFDA guidelines for bioanalytical method validation. A linear response was observed over the range of $2-4100 \mathrm{ng} / \mathrm{mL}$ for Pioglitazone $1-1800 \mathrm{ng} / \mathrm{mL}$ for hydroxy pioglitazone and $3-6400 \mathrm{ng} / \mathrm{ml}$ for Glimepiride with satisfactory precision and accuracy. The lower limit of quantification (LLOQ) was 2, 1 and $3 \mathrm{ng} / \mathrm{Ml}$ for Pioglitazone, hydroxy pioglitazone and Glimepiride respectively. The percent relative error (\%RE) of accuracy studies at three quality control levels was in the range of -3.33 to $3.60 \%$ for PIO, 0.35 to $0.55 \%$ for OH-PIO and -0.83 to $0.53 \%$ for GLM at Intra-day and inter-day precision studies respectively. The precision \%RSD values were less than $4.01 \%$ for PIO, 5.23\% for OH-PIO and 1.78\% for GLM at overall comparison of both intra-day and inter-day studies. The method was reproducible and sensitive enough to quantitative Pioglitazone, hydroxy pioglitazone and Glimepiride in rat plasma samples of a preclinical pharmacokinetic study. Due to the potential scope of pioglitazone-Glimepiride combination to be therapeutically explored, this method is expected to have significant usefulness in future. Keywords: Pioglitazone; Glimepiride; Hydroxy Pioglitazone; Method Validation; LC-MS/MS; Rat Plasma; Pharmacokinetic Applicability 


\section{Introduction}

Type II diabetes mellitus is a heterogeneous metabolic disorder characterized by two important defects, typically in both insulin secretion and insulin sensitivity $[1,2]$. The insulin resistance is well established early in the natural history of type II diabetes [1-3], but with time there is a progressive failure of $\beta$-cell function $[1,4,5]$. Oral hypoglycemic agents generally target one or more events of the pathophysiology and once initial monotherapy starts to lose its effect, long-term management with combination therapy with two oral agents is often necessary [6]. Based on the pathophysiology and pharmacology of type II diabetes, combination therapy with an insulin secretagogue and an insulin sensitizer serves as better therapeutic approach to reduce blood glucose levels in poorly controlled type II diabetic patients [7]. After the introduction of thiazolidinediones, a class of insulin-sensitizing agents, for the treatment of type II diabetes [8], and the choice of Pioglitazone( $( \pm)$ 5-(\{4-[2-(5-ethylpyridin-2-yl)ethoxy]phenyl $\}$ methyl)-1,3-thiazolidine-2,4-dione (Figure 1A), a member of thiazolidinediones class, as a therapeutic agent, improves hyperglycemia, reduces hyperinsulinemia and ameliorates hypertriglyceridemia in a variety of insulin-resistant animal models of impaired glucose tolerance $[9,10]$. Pioglitazone (PIO) selectively activates peroxisome proliferator-activated receptor- $\alpha$ (PPAR- $\alpha$ ) and increases insulinstimulated glucose uptake in peripheral tissues [11] as well as insulin sensitivity in hepatic and adipose tissues [12-14]. PIO is extensively metabolized by hydroxylation and oxidation; Metabolites M-II and M-IV (hydroxy derivatives of Pioglitazone) (Figure 1C) and M-III (keto derivative of pioglitazone) are pharmacologically active $[15,16]$. Glimepiride, (3-ethyl-4-methyl-N-\{2-[4-(\{[(4-methylcyclohexyl)carbamoyl] amino\} sulfonyl)phenyl] ethyl\}-2-oxo-2, 5-dihydro-1H-pyrrole-1-carboxamide, (Figure 1C) (GLM) is alongacting sulfonylurea that inhibits ATP-sensitive potassium channels and stimulates secretion of insulin from pancreatic $\beta$-cells into the blood [17]. Concomitant use of Glimepiride as insulin secretagogue and Pioglitazone as an insulin sensitizer exerts a synergistic action and should achieve better control of blood glucose levels.

Although quantitative determination of each single analyte (PIO alone or GLM alone) [18-23], and limited studies of simultaneous quantification of PIO and OH-PIO [24,25] by LC-MS/MS has been reported, analytical methods that allow simultaneous detection of PIO, OH-PIO and GLM in any source of biological fluid has not been reported yet. Therapeutic drug monitoring for the treatment for Type II diabetic patients necessitates the measurement of the<smiles>CCc1ccc(CCOc2ccc(CC3SC(=O)NC3=O)cc2)nc1</smiles>

Figure 1A: Pioglitazone (PIO).<smiles>CC(O)c1ccc(CCOc2ccc(CC3SC(=O)NC3=O)cc2)nc1</smiles>

Figure 1B: Hydroxy pioglitazone (OH-PIO).

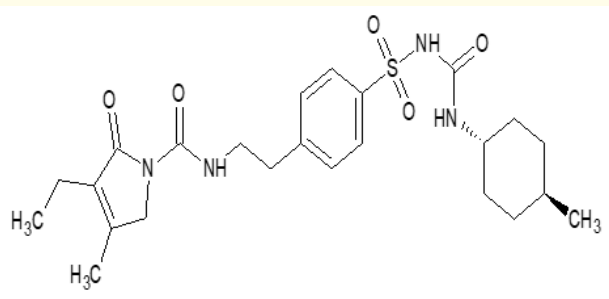

Figure 1C: Glimepiride (GLM).

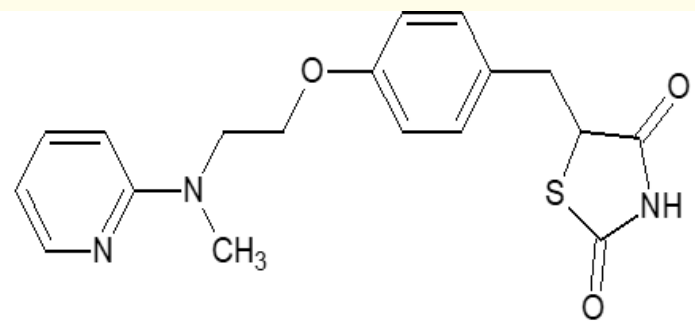

Figure 1D: Rosiglitazone (IS). 
Development and Validation of Highly Selective LC-MS/MS Method for the Simultaneous Estimation of Pioglitazone, Hydroxy Pioglitazone and Glimepiride in in Rat Plasma

plasma concentrations of PIO, OH-PIO and GLM for studying the pharmacokinetics of these drugs and assessment of bioequivalence of commercially available tablet formulation and test formulation for optimization of dosing in combination therapy [26]. Therefore, a sensitive, reliable and rapid method to quantify simultaneously PIO, OH-PIO and GLM in rat plasma is required as an initial study. In this method, a simple, accurate and highly precise method was developed for the simultaneous determination of PIO, OH-PIO and GLM in rat plasma using LC-MS/MS following the USFDA guidelines in presence of Rosiglitazone (Figure1D), an internal standard. Sensitivity and selectivity were enhanced by using an MRM detector coupled with an acidic mobile phase, rather than UV detection. To the best of our knowledge, this simultaneous estimation of Pioglitazone, hydroxyl pioglitazone and Glimepiride in rat plasma could be successfully applied to a rat pharmacokinetic study following gastric administration of PIO and GLM. This method successfully provides a practical, sensitive, and simultaneous LC-MS/MS method applicable for use in clinical research, after being investigated in human plasma.

\section{Materials and Methods}

\section{Materials and reagents}

The reference samples of Pioglitazone (99.63\%), hydroxy pioglitazone (98.83\%), Glimepiride (98.97\%) were procured from Prudence Pharmachem, Ankleshwar, Gujarat, India and Rosiglitasone (99.46\%) used as an internal standard (IS) in this study, was obtained from Hetero drugs Ltd, Hyderabad, India. HPLC grade acetonitrile, methanol and analytical grade ammonium formate were purchased from Merck Specialties Pvt. Ltd., Mumbai, India. All aqueous solutions including the buffer for the mobile phase were prepared with Milli-Q (Millipore, Milford, MA, USA) grade water.

Instrumentation and chromatographic conditions

An HPLC system (Shimadzu, Kyoto, Japan) consisting of a Zorbax SB C18 column ( $50 \mathrm{~mm} \times 4.6 \mathrm{~mm}, 3.5 \mu \mathrm{m}$; Agilent Technologies), a binary LC-20 AD prominence pump, an auto sampler (SIL-HTc) and a solvent degasser (DGU-20 $\mathrm{A}_{3}$ ) were used for the analytical study. Aliquots of the processed samples $(10 \mu \mathrm{L})$ were injected into the column, which was kept at ambient temperature $\left(23 \pm 2^{\circ} \mathrm{C}\right)$. An isocratic mobile phase consisting of an 80:20 (v/v) mixture of acetonitrile and $10 \mathrm{mM}$ ammonium formate $(\mathrm{pH}: 3.0 \pm 0.05)$ was used to separate the analytes and delivered at a flow rate of $0.8 \mathrm{~mL} / \mathrm{min}$ into the electrospray ionization chamber of the mass spectrometer. Quantitation was achieved with MS-MS detection in positive ion mode for both the analytes and the internal standard using an MDS Sciex API- 4000 mass spectrometer equipped with a Turboionspray $\mathrm{TM}$ interface at $550^{\circ} \mathrm{C}$. The ion spray voltage was set at $4800 \mathrm{~V}$. The source parameters i.e., the nebulizer gas, curtain gas, auxiliary gas and collision gas were set at 33, 15, 35 and 7 psi, respectively. The compound parameters i.e. the declustering potential (DP), collision energy (CE), entrance potential (EP) and collision cell exit potential (CXP) were 62, 38, 10 and $3 \mathrm{~V}$ for Pioglitazone and Hydroxy pioglitazone; 70, 34, 10, and $6 \mathrm{~V}$ for Glimepiride; 30, 46, 4, $15 \mathrm{~V}$ for Rosiglitazone (IS).

Detection of the ion pairs were performed by multiple reaction monitoring (MRM) mode. The $\mathrm{m} / \mathrm{z}$ transition pairs (precursor ion/product ion) 357.0/134.1 for PIO, 373.0/150.1 for $\mathrm{OH}$ PIO, 491.2/352.2 for GLM and 358.3/135.2 for IS (Figure 2) PIO $\left(\mathrm{C}_{19} \mathrm{H}_{20} \mathrm{~N}_{2} \mathrm{O}_{3} \mathrm{~S}\right)$ shows its protonated parent ion at $357(\mathrm{~m}+\mathrm{H})$. The product ion $\left(\mathrm{C}_{9} \mathrm{H}_{12} \mathrm{~N}^{+}, 134.1\right)$ for PIO forms after complete cleavage of the molecule at its ether bond. OH-PIO $\left(\mathrm{C}_{19} \mathrm{H}_{20} \mathrm{~N}_{2} \mathrm{O}_{4} \mathrm{~S}\right)$ shows its protonated parent ion at $373.0(\mathrm{~m}+\mathrm{H})$ and the product ion $\left(\mathrm{C}_{9} \mathrm{H}_{12} \mathrm{O}_{1} \mathrm{~N}^{+}\right.$,150.1) for OH-PIO forms after cleavage of the parent molecule at its ether linkage. GLM $\left(\mathrm{C}_{24} \mathrm{H}_{34} \mathrm{~N}_{4} \mathrm{O}_{5} \mathrm{~S}\right)$ shows its protonated ion at $491.2(\mathrm{~m}+\mathrm{H})$ and the product ion $\left(\mathrm{C}_{17} \mathrm{H}_{20} \mathrm{~S}_{1} \mathrm{O}_{5} \mathrm{~N}_{3}{ }^{+}, 352.2\right)$, for GLM generates as result of molecular cleavage at amide linkage adjoining p-methyl cyclohexylamine fragment. Rosiglitazone $\left(\mathrm{C}_{18} \mathrm{H}_{19} \mathrm{~N}_{3} \mathrm{O}_{3} \mathrm{~S}\right)$ shows its protonated parent ion at $358.3(\mathrm{~m}+\mathrm{H})$. The product ion $\left(\mathrm{C}_{8} \mathrm{H}_{11} \mathrm{~N}_{2}{ }^{+}, 135.2\right)$ forms similar way after cleavage of its ether bond as in the case of PIO and OH-PIO. Quadrupoles Q1 and Q3 were set on unit resolution. The analysis data obtained were processed by Analyst software ${ }^{\mathrm{TM}}$ (version 1.4.2).

Preparation of stock and working solutions of analytes and IS

The primary stock solutions of PIO, OH-PIO, GLM and IS were prepared in acetonitrile at $1 \mathrm{mg} / \mathrm{mL}$, separately and these stocks were stored at $2-8^{\circ} \mathrm{C}$; they were found to be stable for 1 month. The stock solutions were suitably diluted with a mixture of acetonitrile and water (80:20, v/v; diluent) to prepare working standard solutions for the purpose of plotting the calibration curve (CC). Appropriate dilutions made working standard solutions of 194.4, 64.8, 21.6, 7.2, 2.4, 0.8, and $0.08 \mu \mathrm{g} / \mathrm{ml}$ for PIO, 97.2, 32.4, 10.8, 3.6, $1.2,0.4$, and $0.04 \mu \mathrm{g} / \mathrm{mL}$ for OH-PIO, 291.6, 97.2, 32.4, 10.8, 3.6, 1.2, 
and $0.12 \mu \mathrm{g} / \mathrm{mL}$ for GLM on day of analysis. Another set of working stock solutions were made in diluent (from primary stock) at 164 (HQC), 52 (MQC), 6 (LQC) and $0.08 \mu \mathrm{g} / \mathrm{mL}$ (LLOQ) for PIO, 72 (HQC), 30 (MQC), 2.8 (LQC) and $0.04 \mu \mathrm{g} / \mathrm{mL}$ (LLOQ) for OH-PIO and 256 (HQC), 92 (MQC), 8.4 (LQC) and $0.12 \mu \mathrm{g} / \mathrm{mL}$ (LLOQ) for GLM for the preparation of QC samples accordingly. A working IS solution $(2 \mu \mathrm{g} / \mathrm{mL})$ was also prepared in diluent. All stock solutions and working solutions were stored at $4{ }^{\circ} \mathrm{C}$ and brought to room temperature before use.

Preparation of calibration and quality control samples

Calibration samples were prepared by spiking $10 \mu \mathrm{L}$ of individual analyte working solution to $320 \mu \mathrm{L}$ of blank rat plasma and adding $50 \mu \mathrm{L}$ of IS solution on the day of analysis to give away final concentrations. All calibration and QC samples were extracted according to method described in sample preparation section. Recovery, precision and accuracy samples were prepared by spiking blank rat plasma with PIO, OH-PIO and GLM at lower limit of quantitation (LLOQ), LQC, MQC and HQC level. Stability study samples were also prepared in the same procedure at LQC and HQC levels and kept at $-20^{\circ} \mathrm{C}$ until analyzed.

\section{Sample preparation}

A $150 \mu \mathrm{L}$ aliquot of rat plasma sample was mixed with $50 \mu \mathrm{L}$ of the internal standard working solution $(2 \mu \mathrm{g} / \mathrm{mL})$. To this, $500 \mu \mathrm{L}$ of $2 \%$ formic acid was added after vortex mixing for 10 s. The sample mixture was loaded onto a Strata-X $33 \mathrm{~mm}$ polymeric sorbent cartridge (30 mg/ $1 \mathrm{~mL}$ ) that was pre-conditioned with $1.0 \mathrm{~mL}$ of methanol followed by $1.0 \mathrm{~mL}$ water. The extraction cartridge was washed with $1.0 \mathrm{~mL}$ of $25 \mathrm{mM}$ ammonium format solution followed by $1.0 \mathrm{~mL}$ of water. PIO, OH-PIO, GLM and IS were eluted with 1.0 $\mathrm{mL}$ of mobile phase. Aliquot of $20 \mu \mathrm{L}$ of the extract was injected into the LC-MS/MS system.

\section{Method validation}

The method was validated for the fundamental validation parameters following the guidelines of US FDA and the International Council for Harmonization (ICH) guidelines [22,23].

\section{Selectivity}

The selectivity or specificity was evaluated by comparing the chromatogram of blank plasma sample (without analytes and IS) from six different batches of rats with those of the blank plasma spiked with analytes at LLOQ level and IS, as well as real plasma samples obtained from rat after administration of PIO and GLM. Any interference from unwanted plasma components at the elution time of PIO, OH-PIO, GLM and IS was evaluated.

Linearity and sensitivity

Linearity was assessed for PIO, OH-PIO and GLM in the concentration range of $2-4860 \mathrm{ng} / \mathrm{mL}(2,20,60,180,540,1620$ and 4860 $\mathrm{ng} / \mathrm{mL})$ for PIO, 1 - $2430 \mathrm{ng} / \mathrm{mL}(1,10,30,90,270,810$, and 2430 $\mathrm{ng} / \mathrm{mL}$ ) for OH-PIO and 3-7290 ng/ml (3, 30, 90, 270, 810, 2430, and $7290 \mathrm{ng} / \mathrm{ml}$ ) for GLM at seven level concentration spiked plasma samples on three separate occasions. The calibration curve was constructed by plotting the peak area ratios (y) of the analytes to IS against the spiked concentrations of the analytes $(\mathrm{x})$ with a $1 / \mathrm{x}^{2}$ weighted linear least squares regression.

\section{Accuracy and precision}

Accurate and precise determination of lower limit of quantification (LLOQ) would define sensitivity of developed method. LLOQ is defined as the lowest concentration of the calibration curve with the signal/noise ratio not less than 10. LLOQ was determined by the analysis of six replicates of LLOQ samples in three separate validation batches. The accuracy of each LLOQ samples should be within $\pm 20 \%$ and the precision should not be greater than $20 \%$.

The intra-day of accuracy and precision were determined by analyzing six replicates of low, medium, high QC samples and LLOQ sample on one occasion. Whereas the inter-day of accuracy and precision were determined by analyzing the four level concentration samples on three consecutive separate occasions and with three separate calibration curves. The relative standard deviation (RSD) was used to assess the precision and the accuracy was expressed by relative errors (RE, \%), respectively.

\section{Extraction recovery and matrix effect}

Extraction recoveries of PIO, OH-PIO, GLM and IS were determined by comparing post extraction and pre extraction spiking samples at low, medium and high QC concentration levels. In post extraction, $150 \mu \mathrm{L}$ blank rat plasma samples were extracted as per the predefined procedure followed by spiking appropriate concentration of analyte solutions into the blank extracts along with the 
IS at $2 \mu \mathrm{g} / \mathrm{mL}$. In pre-extraction, similar concentration levels were spiked in to $150 \mu \mathrm{L}$ blank plasma samples prior to extraction. The samples were prepared and analyzed in six replicates. Extraction recoveries were calculated based on the peak areas of the analytes and IS from regular extracted QC samples to the mean area of the analytes and IS from blank extracts spiked after extraction.

The matrix effect of PIO and GLM was tested in six different sources of rat plasma at three QC concentration levels (low, medium and high), respectively. It was evaluated by the normalized matrix factors, the peak area ratio of analytes, and corresponding IS in post-processed spiked samples to that in non-processed samples. The recovery of IS was evaluated at a final concentration.

\section{Stability}

The stability of the analytes were evaluated by analyzing low and high concentration QC samples in six replicates which were exposed to different storing and handling conditions. For shortterm and long-term stability, QC samples were exposed at room temperature for $24 \mathrm{~h}$ and stored at $-20^{\circ} \mathrm{C}$ for 14 days, respectively. Freeze-thaw stability was assessed by analyzing samples through three freeze-thaw cycles, namely defrosted unassisted at room temperature and refrozen in a freezer at $-20^{\circ} \mathrm{C}$ for three times.

\section{Results and Discussion}

\section{Method development}

Optimization of sample preparation

An efficient sample preparation process could achieve a desired recovery and eliminate the interference from endogenous components from the matrix. Reported methods have employed Liquid-Liquid extractions (LLE) techniques to extract the PIO and GLM from different animal sources of plasma including human. Initial attempts of LLE method using various solvents like dichloromethane, ethyl acetate, methyl t-butyl ether were failed in successful extraction of hydroxy pioglitazone (OH-PIO), Key metabolite of Pioglitazone from spiked blank plasma samples unlike PIO and GLM isolation. As a purpose to develop a novel and efficient procedure to extract OH-PIO along with PIO and GLM, Solid phase extraction (SPE) technique was employed to extract analytes of interest. Moreover, SPE give much cleaner extracts with minimal or no matrix effect. Among the different cartridges, StarataX polymeric sorbent, Oasis HLB, Bond ElutPlexa and Orpheus C18 extraction cartridges tested, StarataX $33 \mathrm{~mm}$ polymeric sorbent cartridges were found to be best for the separation of desired analytes. The recovery for the analytes and the IS were good and reproducible. During the washing step, the cartridges were washed with $25 \mathrm{mM}$ ammonium formate solution to remove the endogenous matrix components efficiently. High structural similarities between Rosiglitazone, PIO and OH-PIO as well as best suitability to receive assay precision and limit variable recovery of all the analytes from plasma sample, rosiglitazone was selected as an internal standard (IS). Hence, Rosiglitazone was used for the quantification of Pioglitazone, Hydroxy pioglitazone and Glimepiride, respectively.

\section{Escalation of MS/MS method}

Optimization of mass spectrometry: In order to optimize ESI conditions for PIO, OH-PIO, GLM and IS, quadrupole full scans were carried out both in positive and negative ion detection mode and found that good response was achieved in positive ionization mode. In the Q1 full scan mode, the protonated precursor $[\mathrm{M}+\mathrm{H}]^{+}$of PIO, OH-PIO, GLM and IS, were $\mathrm{m} / \mathrm{z}$ 357.0, 373.0, 491.2 and 358.3 respectively. Then in the $\mathrm{MS}_{2}$ scan mode, the ion $\mathrm{m} / \mathrm{z} 134.1,150.1$, 352.2 and 135.2 were selected as product ions of PIO, OH-PIO, GLM and IS, respectively. Therefore, the ion transitions monitored for quantification were $\mathrm{m} / \mathrm{z} 357.0 / 134.1$ for PIO, m/z 373.0/150.1 for OH-PIO, m/z 491.2/352.2 for GLM and 358.3/135.2 for IS.

(I)

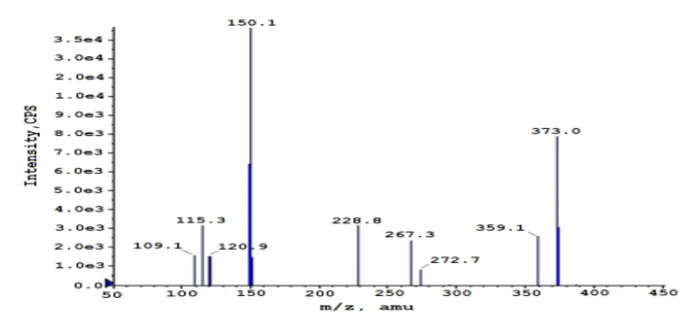

(II)

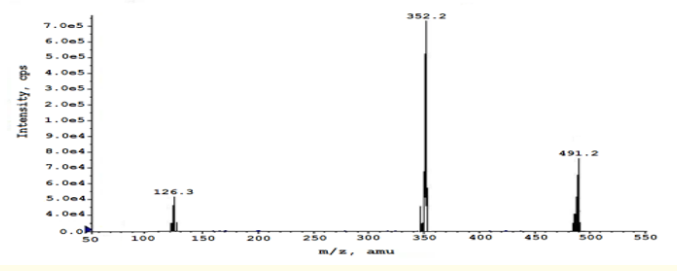

Citation: Kalyani Lingamaneni and Mukthinuthalapati Mathrusri Annapurna. "Development and Validation of Highly Selective LC-MS/MS Method for the Simultaneous Estimation of Pioglitazone, Hydroxy Pioglitazone and Glimepiride in in Rat Plasma". Acta Scientific Pharmaceutical Sciences 5.9 (2021): 05-16. 
(III)

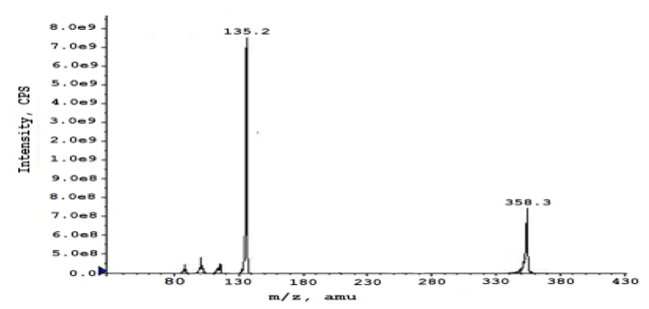

(IV)

Figure 2: Product ion mass spectra of (I) Pioglitazone (II) Hydroxy Pioglitazone (III) Glimepiride and (IV) Rosiglitazone IS.

\section{Optimization of liquid chromatography}

A common and practical Zorbax SB C18 column (50 mm x 4.6 $\mathrm{mm}, 3.5 \mu \mathrm{m}$; Agilent Technologies) was used in our study, and a precolumn Eclipse XDB was applied for protecting the column from biological matrix. In order to separate all analytes and IS with a good resolution, the composition and proportion of the mobile phase was investigated. The results indicated that an isocratic elution with a mobile phase consisting of an 80:20 (v/v) mixture of acetonitrile and $10 \mathrm{mM}$ ammonium formate $(\mathrm{pH}: 3.0 \pm 0.05)$ was provided the best resolution of all analytes as well as IS. The flow rate was $0.8 \mathrm{~mL} / \mathrm{min}$. The analytes and IS were eluted for a short time, as $1.93 \mathrm{~min}, 2.27 \mathrm{~min} 2.84$ and $1.69 \mathrm{~min}$ for Pioglitazone, Hydroxy pioglitazone, Glimepiride and Rosiglitazone, respectively. So, we cut down the time to $5 \mathrm{~min}$, and first few minutes adopted an isocratic elution, and then change the proportion of the mobile phase for washing and balancing the column.

\section{Validation of analytical method}

\section{Selectivity}

The degree of interference by endogenous plasma constituents with the analytes of study and the IS was assessed by inspection of MRM chromatograms of a blank plasma sample, a plasma sample spiked with PIO, OH-PIO, GLM at LLOQ and IS, and a plasma sample from SD rat $1 \mathrm{~h}$ after intragastric administration of PIO, GLM are shown in figure 3. No significant interfering peaks from endogenous compounds are observed at the retention times of analytes and IS. The method was found capable to separate and quantify PIO, OH-PIO and GLM in the presence of other plasma components.

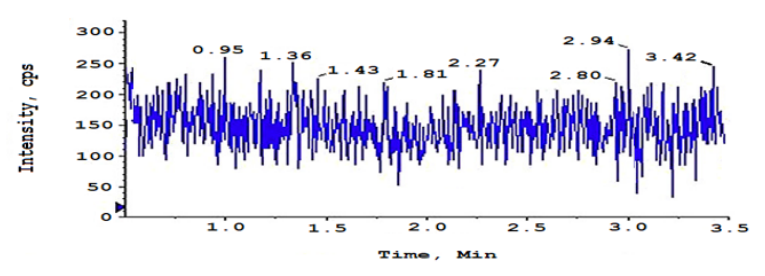

Figure 3AI: MRM chromatograms of PIO in blank plasma sample.

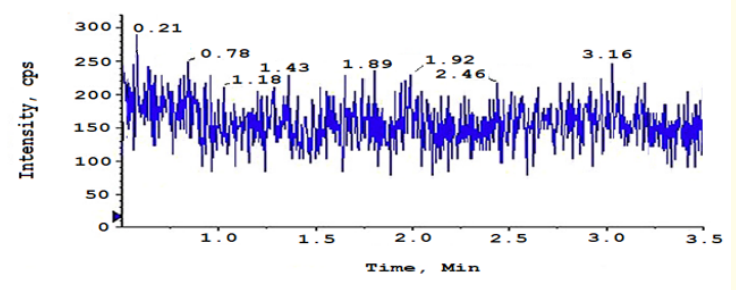

Figure 3AII: MRM chromatograms of OH-PIO in blank plasma sample.

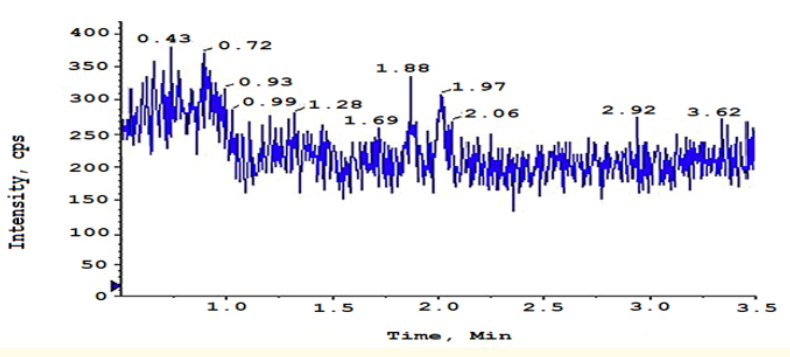

Figure 3AIII: MRM chromatograms of GLM in blank plasma sample. 


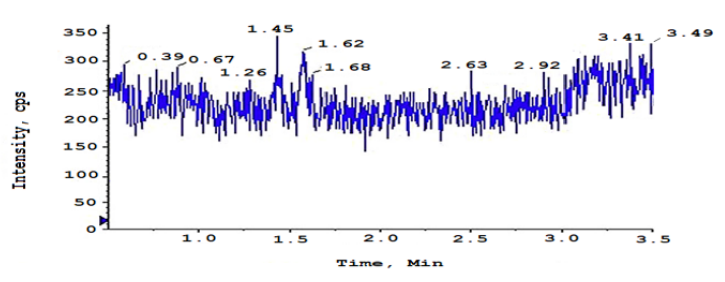

Figure 3AIV: MRM chromatograms of IS in blank plasma sample.

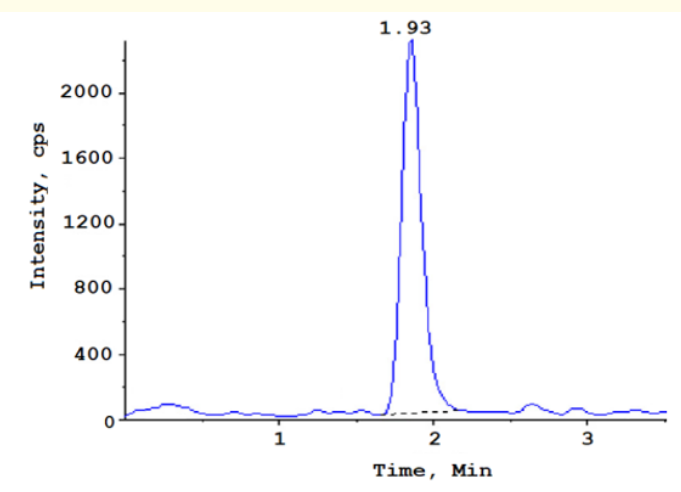

Figure 3BI: MRM chromatograms of PIO in plasma extracted sample at LLOQ.

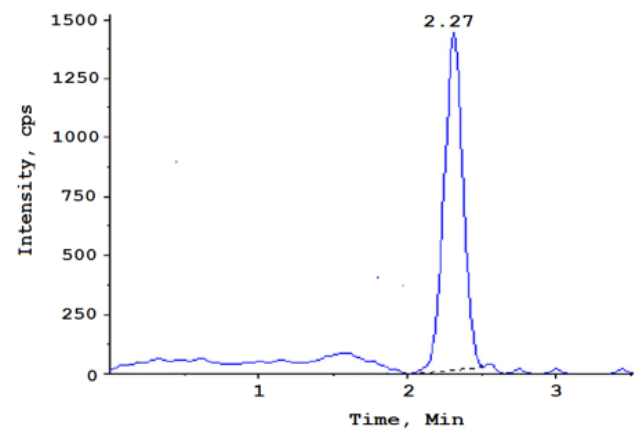

Figure 3BII: MRM chromatograms of OH-PIO (II), in plasma extracted sample at LLOQ.

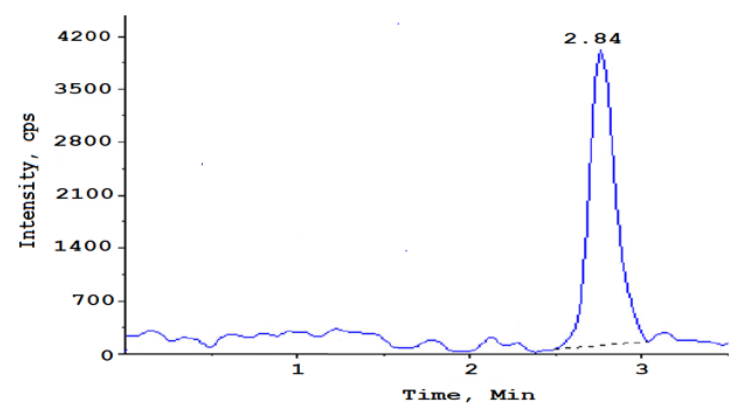

Figure 3BIII: MRM chromatograms of GLM (III) in plasma extracted sample at LLOQ.

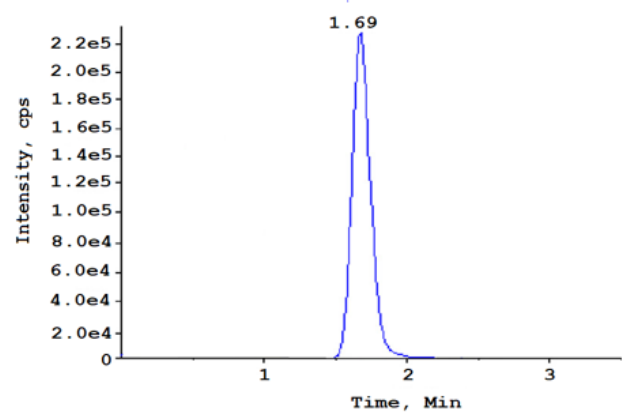

Figure 3BIV: MRM chromatograms of IS (IV) in plasma extracted sample at LLOQ.

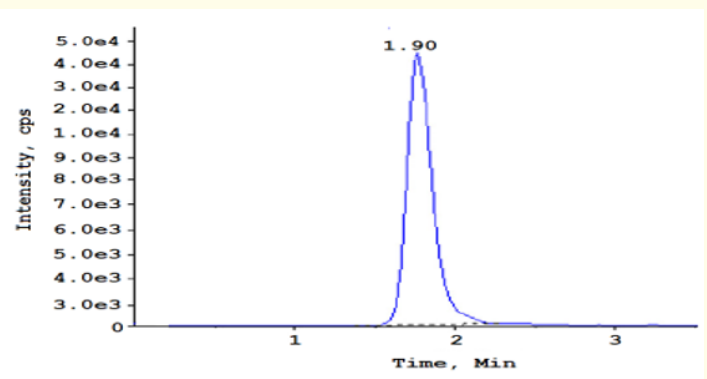

Figure 3CI: MRM chromatograms of PIO in extracted plasma sample at $1 \mathrm{~h}$ after combined administration of PIO and GLM. 


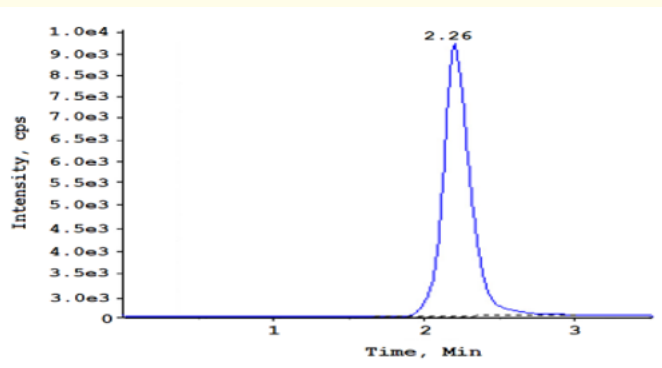

Figure 3CII: MRM chromatograms of OH-PIO in extracted plasma sample at $1 \mathrm{~h}$ after combined administration of PIO and GLM.

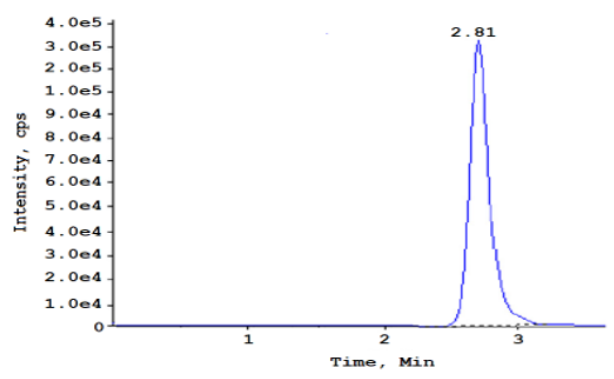

Figure 3CIII: MRM chromatograms of GLM (III) in extracted plasma sample at $1 \mathrm{~h}$ after combined administration of PIO and GLM.

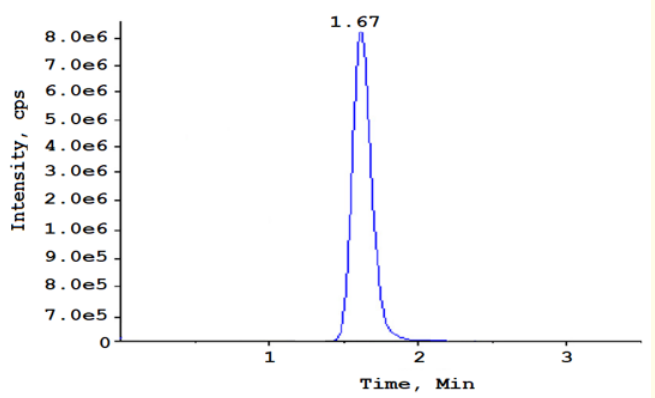

Figure 3CIV: MRM chromatograms of IS in extracted plasma sample at $1 \mathrm{~h}$ after combined administration of PIO and GLM.
Linearity and sensitivity

The plasma calibration curves were linear $\left(r^{2}>0.999\right)$ over the concentration range of $2-4,860 \mathrm{ng} / \mathrm{mL}$ for PIO, 1-2,430 ng/mL for $\mathrm{OH}-\mathrm{PIO}$ and 3-7,290 ng/mL for GLM. The standard curve had a reliable reproducibility over the standard concentrations across the calibration range. Calibration curve was prepared by determining the best fit of peak-area ratios (peak-area of analyte/peak-area of IS) versus concentration of analyte and fitted to the $\mathrm{y}=\mathrm{mx}+$ c. The regression equations were $y=0.010 x+0.103$ for PIO and $y=0.011 x+0.153$ for OH-PIO, $y=0.010 x+0.178$ for GLM. The average regression $(\mathrm{n}=3)$ for PIO, OH-PIO and GLM was found to be $>0.9990,0.9993$ and 0.9991 , respectively. The number of standards used in constructing the standard curve was sufficient to establish the analyte/response relationship.

The lowest concentration with the RSD $<20 \%$ was taken as LLOQ and was found to be $2 \mathrm{ng} / \mathrm{mL}$ for PIO and $1 \mathrm{ng} / \mathrm{mL}$ for $\mathrm{OH}$ PIO and $3 \mathrm{ng} / \mathrm{ml}$ for GLM. The accuracy and precision were expressed in \%RSD and \%RE respectively. The precision of analytes at LLOQ was found to be $4.01 \%, 4.23 \%$ and $0.92 \%$ and the accuracy was $-3.33 \%,-3.51 \%,-0.83 \%$ for PIO, OH-PIO and GLM respectively.

\section{Accuracy and precision}

The results of accuracy and precision over LOQ MOQ, HOQ and LLOQ samples are shown in table 1 . The accuracy was in the range of -3.33 to $3.60 \%$ for PIO, 0.35 to $0.55 \%$ for OH-PIO and -0.83 to $0.53 \%$ for GLM at Intra-day and inter-day precision studies respectively. The precisions were less than $4.01 \%$ for PIO, $5.23 \%$ for $\mathrm{OH}$ PIO and $1.78 \%$ for GLM at overall comparison of both intra-day and inter-day studies. These results of accuracy, precision and dilution integrity met the acceptable criteria for bio analytical method validation.

\section{Extraction recovery and matrix effect}

The absolute recovery data for PIO, OH-PIO and GLM are shown in table 2. Recovery was found to be in the range 99.77-101.74\% for PIO and 98.68-100.83\% for OH-PIO, 99.53-102.03\% for GLM. After the extraction procedures, the recoveries were consistent and precise between analytes IS and the mean extraction recovery of IS was $99.21 \pm 1.3 \%(n=18)$. The results showed the developed method had high extraction efficiency and the recovery was not concentration dependent. 
Development and Validation of Highly Selective LC-MS/MS Method for the Simultaneous Estimation of Pioglitazone, Hydroxy Pioglitazone and Glimepiride in in Rat Plasma

\begin{tabular}{|c|c|c|c|c|c|c|c|c|}
\hline Analyte & $\begin{array}{c}\text { Measurement } \\
\text { Level }\end{array}$ & $\begin{array}{l}\text { Nominal } \\
\text { Conc. } \\
(\mathrm{ng} / \mathrm{mL})\end{array}$ & \multicolumn{3}{|c|}{ Intra-day $(n=6)$} & \multicolumn{3}{|c|}{ Inter-day $(n=18)$} \\
\hline \multirow{3}{*}{ PIO } & LQC & 150 & $150.94 \pm 0.82$ & 0.54 & 0.63 & $149.79 \pm 0.43$ & 0.28 & -0.13 \\
\hline & MQC & 1300 & $1295.51 \pm 1.49$ & 0.11 & -0.34 & $1298.57 \pm 1.04$ & 0.08 & -0.10 \\
\hline & HQC & 4100 & $4097.95 \pm 1.92$ & 0.04 & -0.04 & $4098.82 \pm 1.10$ & 0.02 & -0.02 \\
\hline OH-PIO & LLOQ & 1 & $0.96 \pm 0.04$ & 4.23 & -3.5 & $1.00 \pm 0.05$ & 5.36 & 0.55 \\
\hline \multirow[t]{4}{*}{ GLM } & LLOQ & 3 & $2.97 \pm 0.02$ & 0.92 & -0.83 & $3.01 \pm 0.05$ & 1.78 & 0.53 \\
\hline & LQC & 210 & $209.60 \pm 0.48$ & 0.23 & -0.18 & $210.14 \pm 0.46$ & 0.22 & 0.06 \\
\hline & MQC & 2300 & $2297.27 \pm 0.82$ & 0.03 & -0.11 & $2298.42 \pm 1.38$ & 0.06 & -0.06 \\
\hline & HQC & 6400 & $6397.37 \pm 1.74$ & 0.02 & -0.04 & $6399.36 \pm 0.81$ & 0.01 & -0.01 \\
\hline
\end{tabular}

Table 1: LLOQ Accuracy, and precision of PIO, OH-PIO and GLM in rat plasma by LC-MS/MS method.

\begin{tabular}{|c|c|c|c|c|c|c|}
\hline \multirow[b]{2}{*}{ Analyte } & \multirow[b]{2}{*}{$\begin{array}{l}\text { Measurement } \\
\text { Level }\end{array}$} & \multirow[b]{2}{*}{$\begin{array}{l}\text { Nominal Conc. } \\
\text { In ng/mL }\end{array}$} & \multicolumn{2}{|c|}{ Extraction Recovery } & \multicolumn{2}{|c|}{ Matrix effect } \\
\hline & & & $\begin{array}{c}\text { Recoveries in \% } \\
\text { (Mean } \pm \text { SD) }\end{array}$ & $\begin{array}{l}\text { RSD } \\
\text { (\%) }\end{array}$ & $\begin{array}{l}\text { IS-Normalized matrix } \\
\text { factor }\end{array}$ & RSD (\%) \\
\hline \multirow[t]{3}{*}{ PIO } & LQC & 150 & $101.00 \pm 2.59$ & 2.56 & 0.98 & 1.12 \\
\hline & MQC & 1300 & $99.77 \pm 1.87$ & 1.88 & 0.99 & 1.52 \\
\hline & $\mathrm{HQC}$ & 4100 & $101.24 \pm 3.76$ & 3.72 & 0.98 & 2.58 \\
\hline \multirow[t]{3}{*}{ OH-PIO } & LQC & 70 & $98.68 \pm 1.73$ & 1.76 & 0.99 & 1.36 \\
\hline & MQC & 750 & $98.81 \pm 1.76$ & 1.78 & 0.98 & 1.66 \\
\hline & HQC & 1800 & $100.83 \pm 0.41$ & 0.41 & 1.03 & 0.45 \\
\hline \multirow[t]{3}{*}{ GLM } & LQC & 210 & $100.38 \pm 1.28$ & 1.28 & 0.98 & 1.28 \\
\hline & MQC & 2300 & $99.53 \pm 1.33$ & 1.34 & 1.00 & 1.34 \\
\hline & HQC & 6400 & $102.03 \pm 0.49$ & 0.48 & 1.02 & 0.48 \\
\hline
\end{tabular}

Table 2: The recoveries and matrix effects of PIO, OH-PIO and GLM in rat plasma by LC-MS/MS method.

The matrix effect was assessed by comparing the signals of analytes in plasma with those of analytes dissolved in mobile phase at the same concentration. Matrix effect was expressed as IS normal- ized matrix factor (Matrix factor $=$ response of post spiked concentrations/response of neat concentrations). Measured IS-normalized matrix factor values at LQC, MQC and HQC of PIO were 0.98, 
Development and Validation of Highly Selective LC-MS/MS Method for the Simultaneous Estimation of Pioglitazone, Hydroxy Pioglitazone and Glimepiride in in Rat Plasma

$0.99,0.98$, and the RSD were $1.12 \%, 1.52 \%, 2.52 \%$ and at LQC, MQC and HQC of OH-PIO were $0.99,0.98,1.03$ and the RSD were $1.36 \%$, $1.66 \%, 0.45 \%$ and at LQC, MQC and HQC of GLM 0.98, 1.00 and 1.02 and the RSD were $1.28 \%, 1.34 \%, 0.48 \%$ respectively. The above results are all within the acceptance limit and it illustrated that the rat plasma matrix had no interference for the analysis of $\mathrm{PIO}, \mathrm{OH}$ PIO and GLM.

\section{Stability}

The stability of the analytes were evaluated by analyzing low and high concentration $\mathrm{QC}$ samples in six replicates which were exposed to different storing and handling conditions. These results (Table 3) were compared with the nominal values and were expressed in RSD (\%) and RE (\%).

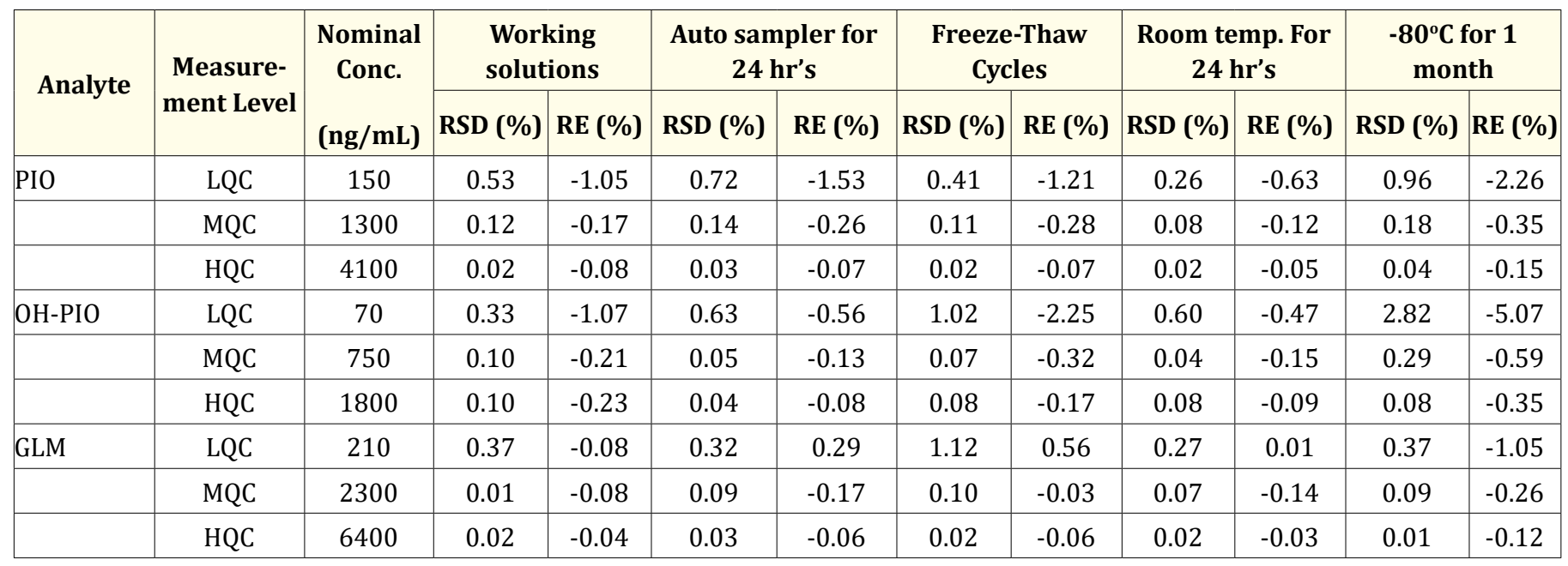

Table 3: Stability of PIO, OH-PIO and GLM under various storage conditions.

\section{Conclusion}

In summary, the method we have developed and validated is a sensitive, specific, and precise LC-MS-MS assay to quantify PIO, OHPIO and GLM simultaneously using a single internal standard. This method meets the requirements and provides a high degree of accuracy, sensitivity and specificity using liquid chromatography and detection by electrospray tandem mass spectrometry. From the results of all the validation parameters, we can conclude that the present method can be useful for bioavailability/bioequivalence studies for simultaneous determination of Pioglitazone, hydroxy pioglitazone and Glimepiride in a combined dosage form of PIO and GLM with desired precision and accuracy.

\section{Acknowledgements}

The authors are grateful to Prudence Pharmachem, Ankleshwar, Gujarat, India and Hetero drugs Ltd, Hyderabad, India for providing the drug substances.

\section{Bibliography}

1. DeFronzo RA. "Pathogenesis of type 2 diabetes: metabolic and molecular implications for identifying diabetes genes". Diabetes Rev. 5 (1997): 177-269.

2. Reaven GM. "Role of insulin resistance in human disease". Diabetes 37 (1988): 595-607.

3. Lillioja S., et al. "Impaired glucose tolerance as a disorder of insulin action. Longitudinal and cross-sectional studies in Pima Indians". The New England Journal of Medicine 318 (1988): 1217-1225.

4. Bailey CJ and Peter JG. "Prospective diabetes study group". Lancet 352 (1998): 837-853.

5. Bennett PH., et al. "Sequential changes in serum insulin concentration during development of non-insulin-dependent diabetes". The Lancet 1 (1989): 1356-1359. 
6. Turner RC., et al. "Glycemic control with diet, sulfonylurea, metformin, or insulin in patients with type 2 diabetes mellitus: progressive requirement for multiple therapies (UKPDS 49). UK Prospective Diabetes Study (UKPDS) Group". JAMA 281 (1999): 2005-2012.

7. Ralph A and DeFronzo MD. "Pharmacologic therapy for type 2 Diabetes mellitus". Annals of Internal Medicine 131 (1999): 281-303.

8. Spiegelman BM. "PPAR-gamma: adipogenic regulator and thiazolidinedione receptor". Diabetes 147 (1998): 507-514.

9. Hofmann C., et al. "Glucose transport deficiency in diabetic animals is corrected by treatment with oral anihyperglycemic agent pioglitazone”. Endocrinology 129 (1991): 1915-1925.

10. Kemnitz JW., et al. "Pioglitazone increases insulin sensitivity, reduces blood glucose, insulin, and lipid levels, and lowers blood pressure, in obese, insulin-resistant rhesus monkeys". Diabetes 43 (1994): 204-211.

11. Miyazaki Y., et al. "Improved glycemic control and enhanced insulin sensitivity in type 2 diabetic subjects treated with pioglitazone”. Diabetes Care 24 (2001): 710-719.

12. Miyazaki Y., et al. "Effect of pioglitazone on circulating adipocytokine levels and insulin sensitivity in type 2 diabetic patients". The Journal of Clinical Endocrinology and Metabolism 89 (2004): 4312-4319.

13. Kawamori R., et al. "Pioglitazone enhances splanchnic glucose uptake as well as peripheral glucose uptake in non-insulin-dependent diabetes mellitus. AD-4833 Clamp-OGL Study Group". Diabetes Research and Clinical Practice 41 (1998): 35-43.

14. Kawaguchi-Suzuki M and Frye RF. "Current clinical evidence on pioglitazone pharmacogenomics". Frontiers in Pharmacology 4 (2013): 147.

15. http://www.rxlist.com/actoplus-met-drug/clinical-pharmacology.htm

16. http://www.fda.gov/downloads/Drugs/ Guidance Compliance Regulatory Information/Guidances/ucm088682.pdf.
17. Ashcroft FM and Rorsman P. "Electrophysiology of the pancreatic beta-cell”. Biophysics and Molecular Biology 54 (1989): 87-143.

18. Xue YJ., et al. "Quantitative determination of pioglitazone in human serum by direct injection high performance liquid chromatography, mass spectrometry an its application to a bioequivalence study". Journal of Chromatography B 795 (2003): 215.

19. Lin ZJ., et al. "Simultaneous determination of pioglitazone and its two active metabolites in human plasma by LC-MS/MS". Journal of Pharmaceutical and Biomedical Analysis 33 (2003): 101-108.

20. Pistos C., et al. "Improved liquid chromatographic tandem mass spectrometric determination and pharmacokinetic study of glimepiride in human plasma". Biomed Chromatography 19 (2005): 394-401.

21. Chakradhar L., et al. "A rapid and highly sensitive method for the determination of glimepiride in human plasma by liquid chromatography-electrospray ionization tandem mass spectrometry: Application to a pre-clinical pharmacokinetic study". Biomedical Chromatography 22 (2008): 58.

22. Salem I., et al. "Determination of glimepiride in human plasma by liquid chromatography-electrospray ionization tandem mass spectrometry". Journal of Chromatography B 799 (2004): 103.

23. Hess C., et al. "Simultaneous identification and validated quantification of 11 oral hypoglycaemic drugs in plasma by electrospray ionisation liquid chromatography-mass spectrometry". Analytical and Bioanalytical Chemistry 400 (2011): 33.

24. Jagadeesh B., et al. "Development and validation of highly selective and robust method for simultaneous estimation of pioglitazone, hydroxypioglitazone and metformin in human plasma by LC-MS/MS: Application to a pharmacokinetic study". Journal of Chromatography B 30 (2013): 136-145.

25. Chinnalalaiah R., et al. "Liquid chromatography and tandem mass spectrometry method for quantitative determination of pioglitazone and its metabolite 5-hydroxy pioglitazone in hu- 
Development and Validation of Highly Selective LC-MS/MS Method for the Simultaneous Estimation of Pioglitazone, Hydroxy Pioglitazone and Glimepiride in in Rat Plasma

man plasma". Annales Pharmaceutiques Françaises 75 (2016):

105-111.

26. Bell DS. "Type 2 diabetes mellitus: what is the optimal treatment regimen?". Journal of Medicine 116 (2004): 23S-29S.

27. US Food and Drug Administration Guidance for Industry. Bioanalytical method validation (2013).

28. International Conference on Harmonization. Validation of analytical procedures: methodology ICH Q2 (R1) (2005).

\section{Volume 5 Issue 9 September2021}

(C) All rights are reserved by Kalyani Lingamaneni and Mukthinuthalapati Mathrusri Annapurna. 